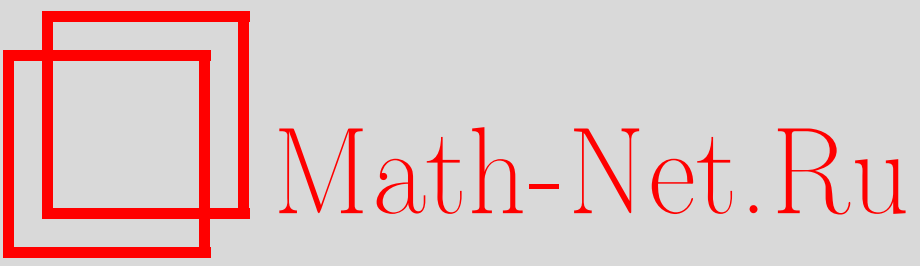

I. Fazekas, Merging to semistable processes, Теория вероятн. и ее примен., 2011, том 56, выпуск 4, 726-741

DOI: https://doi.org/10.4213/tvp4420

Использование Общероссийского математического портала Math-Net.Ru подразумевает, что вы прочитали и согласны с пользовательским соглашением

http://www.mathnet.ru/rus/agreement

Параметры загрузки:

IP : 3.85 .7 .115

26 апреля 2023 г., $17: 46: 07$

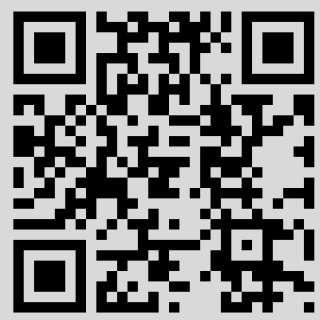




\title{
MERGING TO SEMISTABLE PROCESSES ${ }^{1)}$
}

\begin{abstract}
Получена функциональная теорема состремления для распределений из области геометрического частичного притяжения полуустойчивого закона.
\end{abstract}

Ключевые слова и фразы: расстояния между вероятностными мерами, плотность, функциональная предельная теорема, полуустойчивый закон, область частичного притяжения, медленно меняющаяся функция.

1. Introduction. The concept of semistable distributions was introduced by Paul Lévy in 1937 (see [17]). The characteristic function of a semistable distribution was obtained by Kruglov [16]. The domain of geometric partial attraction of a semistable law was described by Grinevich and Khokhlov [13]. Csörgö and Megyesi in [7] studied the theory of semistable laws in the framework of the «probabilistic» approach of Csörgő [5] and Csörgö, Haeusler, and Mason [6]. In the case of distributions being in the domain of geometric partial attraction of a semistable law ordinary convergence in distribution takes place only along some subsequences. However, a merge theorem is valid [7]. We say that two sequences $\left\{\mu_{n}\right\}$ and $\left\{\nu_{n}\right\}$ of probability measures are merging if $\lim _{n \rightarrow \infty} \varrho\left(\mu_{n}, \nu_{n}\right)=0$, where $\varrho$ is a distance of probability measures (the Lévy distance or the Prokhorov distance, say).

A considerable part of probability theory is devoted to functional versions of ordinary limit theorems (the standard references are [19], [21], [2]). The classical result is Donsker's theorem. Let $\xi_{1}, \xi_{2}, \ldots$ be independent and identically distributed random variables with $\mathbf{E} \xi_{1}=0,\left(\mathbf{D} \xi_{1}\right)^{2}=1$. Denote by $X_{n}(t)$ the usual step function constructed from the partial sums. Then $X_{n} \stackrel{d}{\longrightarrow} W$ in $\mathbf{D}[0,1]$, where $W$ is the standard Wiener process. However, if we assume that $\xi_{1}, \xi_{2}, \ldots$ belong to the domain of geometric partial attraction of a semistable law, then the step functions constructed from $\xi_{1}, \xi_{2}, \ldots$

\footnotetext{
*Department of Applied Mathematics and Probability Theory, University of Debrecen, P.O. Box 12, 4010 Debrecen, Hungary; e-mail: fazekasi@inf.unideb.hu

1) Supported by the Hungarian Foundation of Scientific Researches under Grant № OTKA T-079128/2009.
} 
will not converge (since the finite dimensional distributions will not converge). Therefore the following question arises. How can we describe the asymptotic behavior of the step functions?

In this paper we prove a functional merge theorem for laws being in the domain of geometric partial attraction of a semistable law (Theorem 2.1). It is the functional version of the merge theorem by Csörgö and Megyesi [7]. The accompanying laws in our theorem are distributions of semistable Lévy processes (random processes with stationary independent increments). As we want to prove the precise functional version of (a part of) Theorem 2 of [7], in the following sections we shall use the same centering and norming constants as the ones in [7].

To handle the concept of merging distributions of stochastic processes, we need some facts about merging probabilities on metric spaces (see [8]). It turns out that in our case both sequences of probabilities are tight.

In Section 2 first some known facts on semistable distributions and their domain of geometric partial attraction are listed. Then the main result is given (Theorem 2.1). Section 3 describes the approximation of sums of independent random variables being in the domain of geometric partial attraction of a semistable law. In Section 4 some general facts on merging probability measures are listed, moreover, tightness of our sequences is proved.

We shall use the following notation. $\mathbf{N}$ is the set of positive integers, $\mathbf{R}$ denotes the set of real numbers. $\mathbf{D}[0,1]$ is the Skorokhod space of functions without discontinuities of second kind (see [2]). The distribution of a random element $\xi$ is denoted by $\mathscr{L}_{\xi}$. Sign $\stackrel{d}{\longrightarrow}$ denotes the convergence in distribution.

Finally, we mention that in [1] an almost sure limit theorem was proved for laws being in the domain of geometric partial attraction of a semistable law. The almost sure limit theorem is valid with the usual weights. In [10] a functional analogue of the almost sure limit theorem of [1] was obtained.

2. The merge theorem. Consider the probabilistic approach to the theory of infinitely divisible distributions presented in [6] and [5]. We shall use it to handle $p$-semistable distributions, $0<p<2$, and the domain of the geometric partial attraction of a $p$-semistable distribution, see [18], [7].

Let $\Psi$ be the class of all nonpositive, nondecreasing, right-continuous functions $g(\cdot)$ defined on the positive half-line $(0, \infty)$ such that $\int_{\varepsilon}^{\infty} g^{2}(s) d s<$ $\infty$ for all $\varepsilon>0$. Let $N_{j}, j=1,2$, be independent standard left-continuous Poisson processes. Let

$$
V_{j}(g)=\int_{1}^{\infty}\left[N_{j}(s)-s\right] d g(s)+\int_{0}^{1} N_{j}(s) d g(s)-g(1), \quad j=1,2 .
$$

Let $U$ be a standard normal random variable such that $N_{1}(\cdot), U$, and $N_{2}(\cdot)$ 
are independent. Let $g_{1}, g_{2} \in \Psi$ and let $\sigma \geqslant 0$. Introduce the random variable

$$
V\left(g_{1}, g_{2}, \sigma\right)=-V_{1}\left(g_{1}\right)+\sigma U+V_{2}\left(g_{2}\right) .
$$

Up to an additive constant, any infinitely divisible distribution can be described in this way.

Infinitely divisible distributions which arise as limiting distributions of suitable centered and normalized sums $S_{k_{n}}=\left(1 / B_{k_{n}}\right) \sum_{i=1}^{k_{n}} X_{i}-A_{k_{n}}$ of independent and identically distributed random variables $X_{1}, X_{2}, \ldots$ along subsequences $\left\{k_{n}\right\}$ satisfying condition $\lim _{n \rightarrow \infty} k_{n+1} / k_{n}=c \geqslant 1$ are called semistable laws. It is known that a semistable law is either normal or $p$ semistable with $0<p<2$.

We consider the case of the $p$-semistable distribution, $0<p<2$. Suppose that

$$
g_{j}(s)=-M_{j}(s) s^{-1 / p}, \quad s>0, \quad j=1,2,
$$

are nondecreasing functions, where $M_{1}, M_{2}$ are nonnegative, rightcontinuous functions on $(0, \infty)$, either identically zero or bounded away from both zero and infinity, such that $M_{1}+M_{2}$ is not identically zero, moreover $M_{j}(c s)=M_{j}(s)$ for all $s>0, j=1,2$, for some constant $c>1$. Let $g_{j}(s)$ be defined by (2.3) and let

$$
\begin{gathered}
W_{j}\left(M_{j}\right)=W_{j}\left(M_{j}, p\right)=V_{j}\left(g_{j}\right), \quad j=1,2, \\
W\left(M_{1}, M_{2}\right)=W_{2}\left(M_{2}\right)-W_{1}\left(M_{1}\right) .
\end{gathered}
$$

A random variable $W$ is a $p$-semistable random variable with $0<p<2$ if and only if $W \stackrel{d}{=} W\left(M_{1}, M_{2}\right)+b$ for some $M_{1}, M_{2}$ and $b \in \mathbf{R}$. We denote by

$$
\psi(x)=\psi\left(x, M_{1}, M_{2}\right)=\mathbf{E} e^{i x W\left(M_{1}, M_{2}\right)}
$$

the characteristic function of $W\left(M_{1}, M_{2}\right)$.

Let $X, X_{i}, i \in \mathbf{N}$, be independent and identically distributed random variables. Denote by $F(x)=\mathbf{P}(X \leqslant x)$ the distribution function of $X$. Let $\left\{k_{n}\right\}$ be a sequence of positive integers with the property

$$
\lim _{n \rightarrow \infty} \frac{k_{n+1}}{k_{n}}=c>1 \text {. }
$$

Recall that $F$ (or $X$ ) is said to belong to the domain of geometric partial attraction of a semistable law, if for some sequence $\left\{k_{n}\right\}$ of positive integers with property (2.6), for some norming numbers $B_{k_{n}}$ and some centering numbers $A_{k_{n}}$, the sequence $S_{k_{n}}=\left(1 / B_{k_{n}}\right) \sum_{i=1}^{k_{n}} X_{i}-A_{k_{n}}$ converges in distribution to $W$ as $n \rightarrow \infty$, where $W$ is a $p$-semistable random variable $\left(W \stackrel{d}{=} W\left(M_{1}, M_{2}\right)\right.$, say). In this case we will write $F \in \mathbf{D}_{\mathrm{gp}}\left(M_{1}, M_{2}, p\right)$, see [18]. Denote by $Q$ the quantile function of $X$, i.e.,

$$
Q(s)=\inf \{x \in \mathbf{R}: F(x) \geqslant s\}, \quad 0<s<1 .
$$


Denote by $Q_{+}$the right-continuous version of the quantile function $Q$.

Consider a subsequence $\left\{k_{n}\right\}_{n=1}^{\infty} \subset \mathbf{N}$ satisfying (2.6). Since $c>1$, the sequence $\left\{k_{n}\right\}$ is eventually strictly increasing. So for all $s \in\left(0, s_{0}\right)$, with $s_{0} \in(0,1]$ small enough, there exists a unique $k_{n^{*}(s)}$ such that $k_{n^{*}(s)}^{-1} \leqslant s<$ $k_{n^{*}(s)-1}^{-1}$. We define $\gamma(s)=s k_{n^{*}(s)}$ for $s \in\left(0, s_{0}\right)$ and $\gamma(s)=1$ for $s \in\left[s_{0}, 1\right)$. So $1 \leqslant \gamma(s)<c+\varepsilon$ for any fixed $\varepsilon>0$ and all $s \in(0,1)$ small enough for the limiting $c>1$ from (2.6). Then $F \in \mathbf{D}_{\mathrm{gp}}\left(M_{1}, M_{2}, p\right)$ along a subsequence $\left\{k_{n}\right\}$ satisfying $(2.6), p \in(0,2)$, if and only if for all $s \in(0,1)$ small enough

$$
\begin{aligned}
Q_{+}(s) & =-s^{-1 / p} l(s)\left[M_{1}(\gamma(s))+h_{1}(s)\right], \\
Q(1-s) & =s^{-1 / p} l(s)\left[M_{2}\left(\gamma(s)+h_{2}(s)\right],\right.
\end{aligned}
$$

where $l(\cdot)$ is a right-continuous function, slowly varying at zero, and the error functions $h_{1}$ and $h_{2}$ are right-continuous such that $\lim _{n \rightarrow \infty} h_{j}\left(t / k_{n}\right)=0$, for every continuity point $t>0$ of $M_{j}, j=1,2$ (see [13], [18]).

We will use the norming and centering constants (see [7])

$$
\begin{aligned}
B_{n} & =n^{1 / p} l\left(\frac{1}{n}\right) \\
A_{n k} & =\frac{k}{B_{n}} \int_{1 / n}^{1-1 / n} Q(s) d s, \quad k=1, \ldots, n, \quad n=1,2, \ldots .
\end{aligned}
$$

Let

$$
S_{n k}=\frac{1}{B_{n}} \sum_{i=1}^{k} X_{i}-A_{n k}, \quad k=1, \ldots, n, \quad n=1,2, \ldots
$$

We will denote $S_{n n}$ and $A_{n n}$ by $S_{n}$ and $A_{n}$, respectively.

Our aim is to obtain a functional version of the following result.

Theorem A (Merge theorem, i.e., Theorem 2 in [7]). Let $F \in$ $\mathbf{D}_{\mathrm{gp}}\left(M_{1}, M_{2}, p\right)$ along a subsequence $\left\{k_{n}\right\}$ satisfying $(2.6), 0<p<2$. Then, as $n \rightarrow \infty$, we have

$$
\sup _{x \in \mathbf{R}}\left|\mathbf{P}\left\{S_{n} \leqslant x\right\}-\mathbf{P}\left\{W\left(M_{1}\left(\gamma\left(\frac{1}{n}\right) y\right), M_{2}\left(\gamma\left(\frac{1}{n}\right) y\right)\right) \leqslant x\right\}\right| \rightarrow 0 .
$$

Here and in what follows $W\left(M_{1}(\gamma(1 / n) y), M_{2}(\gamma(1 / n) y)\right)$ denotes $W\left(\widetilde{M}_{1}, \widetilde{M}_{2}\right)$ with $\widetilde{M}_{1}(y)=M_{1}(\gamma(1 / n) y)$ and $\widetilde{M}_{2}(y)=M_{2}(\gamma(1 / n) y)$.

To obtain the functional version of Theorem A, consider the random processes

$$
Z_{n}(t)=S_{n[n t]}, \quad t \in[0,1],
$$

with sample paths in the Skorokhod space $\mathbf{D}[0,1]$. 
Also we will consider the Lévy processes $Y_{n}(t), t \in[0,1]$, such that

the distribution of $Y_{n}(1)$ is the same as

$$
\text { that of } W\left(M_{1}\left(\gamma\left(\frac{1}{n}\right) y\right), M_{2}\left(\gamma\left(\frac{1}{n}\right) y\right)\right) \text {. }
$$

(For the definition of a Lévy process see [20, Definition 1.6].) By Corollary 11.6 of [20], there exists a Lévy process with property (2.12). In particular, the trajectories of $Y_{n}$ belong to $\mathbf{D}[0,1]$.

Theorem 2.1. Let $F \in \mathbf{D}_{\mathrm{gp}}\left(M_{1}, M_{2}, p\right)$ along a subsequence $\left\{k_{n}\right\}$ satisfying (2.6), $0<p<2$. Let $Z_{n}$ be defined by (2.10)-(2.11) and let $Y_{n}$ be the Lévy process given by (2.12). Then, as $n \rightarrow \infty$,

$$
\varrho\left(\mathscr{L}_{Z_{n}}, \mathscr{L}_{Y_{n}}\right) \rightarrow 0 \quad \text { in } \quad \mathbf{D}[0,1]
$$

P r o o f. By Lemma 4.1, we have to show that $\left\{\mathscr{L}_{Z_{n}}, n=1,2, \ldots\right\}$ and $\left\{\mathscr{L}_{Y_{n}}, n=1,2, \ldots\right\}$ are tight, moreover, the distances of the finite dimensional distributions converge to 0 . These facts are proved in Lemmas 4.3, 4.2, and 3.1 .

3. Approximation with semistable distributions. Let the sequences of processes $Z_{n}$ and $Y_{n}$ be defined by (2.11) and (2.12), respectively. In this section we prove the convergence of the finite dimensional distributions. Moreover, we present the distribution of $Y_{n}$ in terms of Lévy's description of infinitely divisible laws.

First we collect some known facts on characteristic functions and infinitely divisible laws.

$\mathrm{R}$ e $\mathrm{m}$ a r k 3.1 (see [4, Chap. 12]). 1. Let $f(t)$ be a continuous, nonvanishing, complex-valued function of the interval $[-T, T]$ with $f(0)=1$. Then there exists a unique (single-valued) continuous complex-valued function $\lambda(t)$ defined on $[-T, T]$ with $\lambda(0)=0$ such that $f(t)=e^{\lambda(t)}$ for $t \in[-T, T]$. Moreover, $[-T, T]$ is replaceable by $(-\infty, \infty)$.

2 . The function $\lambda(t)$ defined above is called the distinguished logarithm of $f(t)$ and is denoted by $\log f(t)$. Also, for $v \in \mathbf{R}, \exp (v \lambda(t))$ is called the distinguished $v$ th power of $f(t)$ and is denoted by $f^{v}(t)$.

3. Let $f, f_{k}, k=1,2, \ldots$, be continuous, nonvanishing, complex-valued functions of the interval $[-T, T]$ with $f(0)=1, f_{k}(0)=1, k=1,2, \ldots$ If $f_{k} \rightarrow f$ uniformly in $[-T, T]$, then $\log f_{k} \rightarrow \log f$ uniformly in $[-T, T]$.

4. For an infinitely divisible characteristic function $\varphi$ we have $\varphi(t) \neq 0$ for $t \in \mathbf{R}$. The product of infinitely divisible characteristic functions is infinitely divisible. (A characteristic function is called infinitely divisible if it belongs to an infinitely divisible law.)

5 . The limit of infinitely divisible laws is infinitely divisible. 
R e m a r k 3.2 (see [15, Theorem 3.1]). Let $\left\{\mu_{n}: n=1,2, \ldots\right\}$ and $\left\{\nu_{n}: n=1,2, \ldots\right\}$ be families of probability measures on the real line with characteristic functions $\left\{\varphi_{n}: n=1,2, \ldots\right\}$ and $\left\{\psi_{n}: n=1,2, \ldots\right\}$, respectively. Let $\left\{\mu_{n}: n=1,2, \ldots\right\}$ be relatively compact. Then $\varrho\left(\mu_{n}, \nu_{n}\right) \rightarrow 0$ if and only if $\varphi_{n}(t)-\psi_{n}(t) \rightarrow 0$ for every $t \in \mathbf{R}$. (Here $\varrho$ denotes the Lévy distance.)

Lemma 3.1. The Lévy distances of the finite dimensional distributions of the sequence $Z_{n}(\cdot)$ and those of $Y_{n}(\cdot)$ converge to 0 , as $n \rightarrow \infty$.

P r o o f. We shall write $W(M(\gamma(1 / n) y))$ instead of $W\left(M_{1}(\gamma(1 / n) y)\right.$, $\left.M_{2}(\gamma(1 / n) y)\right)$.

Both of the processes $Z_{n}(\cdot)$ and $Y_{n}(\cdot)$ have independent increments and their values at zero are zero. So it is enough to prove that the distances of the distributions of the increments converge to 0 .

Let $0 \leqslant t_{0}<t_{1} \leqslant 1$ and let $\Delta t=t_{1}-t_{0}$. We shall use Remark 3.2. So first we shall prove that the set of distributions of the increments on the interval $\left[t_{0}, t_{1}\right]$ of the family of processes $Y_{n}(\cdot)$ is a relatively compact set of probabilities.

Let $\varphi_{n}=\varphi_{W(M(\gamma(1 / n) y))}$ be the characteristic function of $W(M(\gamma(1 / n) y))$. Then the characteristic function of the increment $Y_{n}\left(t_{1}\right)-Y_{n}\left(t_{0}\right)$ is $\varphi_{n}^{\Delta t}$. We have to prove that every subsequence $\varphi_{n^{\prime}}^{\Delta t}$ contains a convergent subsequence. By Theorem 1 of [7] the distributions of the sequence $\left\{S_{n}\right\}_{n=1}^{\infty}$ is relatively compact. So, by Theorem A, the sequence $W(M(\gamma(1 / n) y)), n=1,2, \ldots$, is also relatively compact. Therefore, the subsequence $\left\{n^{\prime}\right\}$ contains a further subsequence $\left\{n^{\prime \prime}\right\}$ such that $\varphi_{n^{\prime \prime}} \rightarrow \varphi_{\infty}$, where $\varphi_{\infty}$ is a characteristic function. Here the convergence is uniform in any bounded interval, and the characteristic functions involved are infinitely divisible. We have to prove that $\varphi_{n^{\prime \prime}}^{\Delta t}\left(x_{0}\right) \rightarrow \varphi_{\infty}^{\Delta t}\left(x_{0}\right)$ for each fixed $x_{0}$. Let $T$ be large enough so that $x_{0} \in[-T, T]$. By Remark 3.1, $\log \varphi_{n^{\prime \prime}}\left(x_{0}\right) \rightarrow \log \varphi_{\infty}\left(x_{0}\right)$ for $x_{0} \in[-T, T]$. Therefore,

$$
\varphi_{n^{\prime \prime}}^{\Delta t}\left(x_{0}\right)=e^{\Delta t \log \varphi_{n^{\prime \prime}}\left(x_{0}\right)} \rightarrow e^{\Delta t \log \varphi_{\infty}\left(x_{0}\right)}=\varphi_{\infty}^{\Delta t}\left(x_{0}\right) .
$$

Therefore, the set of the distributions of the increments of the processes $Y_{n}(\cdot)$ on the interval $\left[t_{0}, t_{1}\right]$ is relatively compact.

Now turn to the process $Z_{n}$. The increment of $Z_{n}$ on the interval $\left[t_{0}, t_{1}\right]$ is

$$
S_{n\left[n t_{1}\right]}-S_{n\left[n t_{0}\right]}=\frac{1}{B_{n}} \sum_{i=\left[n t_{0}\right]+1}^{\left[n t_{1}\right]} X_{i}-l_{n} A_{n 1},
$$

where $l_{n}=\left[n t_{1}\right]-\left[n t_{0}\right]$. We have $l_{n} / n \rightarrow \Delta t$. The characteristic function of the increment is

$$
\left(\psi_{n}(x)\right)^{l_{n}}=\left(\varphi_{X}\left(\frac{x}{B_{n}}\right) e^{i x A_{n 1}}\right)^{l_{n}}
$$


where $\varphi_{X}$ is the characteristic function of $X$. By Remark 3.2, we have to prove that

$$
\psi_{n}^{l_{n}}(x)-\varphi_{n}^{\Delta t}(x) \rightarrow 0
$$

for every $x \in \mathbf{R}$. Assume that it is not true, therefore there exist a fixed $x_{0} \in \mathbf{R}$, a positive $\varepsilon$, and a subsequence $\left\{n^{\prime}\right\}$ such that

$$
\left|\psi_{n^{\prime}}^{l_{n^{\prime}}}\left(x_{0}\right)-\varphi_{n^{\prime}}^{\Delta t}\left(x_{0}\right)\right|>\varepsilon
$$

for each $n^{\prime}$. Since the sequence $W(M(\gamma(1 / n) y)), n=1,2, \ldots$, is relatively compact, we have a further subsequence $\left\{n^{\prime \prime}\right\}$ of $\left\{n^{\prime}\right\}$ such that

$$
\varphi_{n^{\prime \prime}}(x) \rightarrow \varphi_{\infty}(x) .
$$

Since in (3.3) the characteristic functions are infinitely divisible, that is, they are nonvanishing, we have

$$
\varphi_{n^{\prime \prime}}^{\Delta t}\left(x_{0}\right) \rightarrow \varphi_{\infty}^{\Delta t}\left(x_{0}\right) .
$$

From Theorem A and (3.3), we have $\psi_{n^{\prime \prime}}^{n^{\prime \prime}}(x) \rightarrow \varphi_{\infty}(x)$. Let $T$ be so large that $x_{0} \in[-T, T]$. The previous convergence is uniform in the interval $[-T, T]$ and the limit is infinitely divisible, so it is nonvanishing. Therefore $\psi_{n^{\prime \prime}}^{n^{\prime \prime}}(x)$ is also nonvanishing if $n^{\prime \prime}$ is large enough and $x \in[-T, T]$. By Remark 3.1, we can take the distinguished logarithm and we have $n^{\prime \prime} \log \psi_{n^{\prime \prime}}(x)=\log \psi_{n^{\prime \prime}}^{n^{\prime \prime}}(x) \rightarrow$ $\log \varphi_{\infty}(x)$. Therefore $\left(l_{n^{\prime \prime}} / n^{\prime \prime}\right) n^{\prime \prime} \log \psi_{n^{\prime \prime}}(x) \rightarrow \Delta t \log \varphi_{\infty}(x)$. It implies that

$$
\psi_{n^{\prime \prime}}^{l_{n^{\prime \prime}}}(x)=e^{l_{n^{\prime \prime}} \log \psi_{n^{\prime \prime}}(x)} \rightarrow e^{\Delta t \log \varphi_{\infty}(x)}=\varphi_{\infty}^{\Delta t}(x) .
$$

As (3.5) is valid for any $x \in[-T, T]$ and we have $x_{0} \in[-T, T]$, therefore (3.5) combined with (3.4), contradicts to (3.2). So (3.1) is valid.

By Remark 3.2, the proof is complete. Lemma 3.1 is proved.

Now we turn to the description of $Y_{n}(\cdot)$ in terms of Lévy's original approach to infinitely divisible distributions. Consider the random variable $V\left(g_{1}, g_{2}, \sigma\right)=-V_{1}\left(g_{1}\right)+\sigma U+V_{2}\left(g_{2}\right)$ from equation (2.2). For any $g \in \Psi$ let

$$
\theta(g)=\int_{0}^{1} \frac{g(s)}{1+g^{2}(s)} d s-\int_{1}^{\infty} \frac{g^{3}(s)}{1+g^{2}(s)} d s .
$$

Then, by Theorem 3 of [6], the characteristic function of

$$
V_{0}\left(g_{1}, g_{2}, \sigma\right)=V\left(g_{1}, g_{2}, \sigma\right)+\theta\left(g_{2}\right)-\theta\left(g_{1}\right)
$$

is

$$
\begin{aligned}
\mathbf{E} e^{i x V_{0}\left(g_{1}, g_{2}, \sigma\right)}=\exp \{ & -\frac{\sigma^{2} x^{2}}{2}+\int_{-\infty}^{0}\left(e^{i x u}-1-\frac{i x u}{1+u^{2}}\right) d L(u) \\
& \left.+\int_{0}^{\infty}\left(e^{i x u}-1-\frac{i x u}{1+u^{2}}\right) d R(u)\right\}
\end{aligned}
$$


for all real $x$, where $L(u)=\inf \left\{s>0: g_{1}(s) \geqslant u\right\}, u<0$, and $R(u)=$ $-\inf \left\{s>0: g_{2}(s) \geqslant-u\right\}, u>0$. Here $L(\cdot)$ is left-continuous and nondecreasing on $(-\infty, 0)$ with $L(-\infty)=0$ and $R(\cdot)$ is right-continuous and nondecreasing on $(0, \infty)$ with $R(\infty)=0$, moreover $\int_{-\varepsilon}^{0} u^{2} d L(u)+\int_{0}^{\varepsilon} u^{2} d R(u)<$ $\infty$ for every $\varepsilon>0$. Conversely, consider two functions $L(\cdot)$ and $R(\cdot)$ with the properties just listed, and choose $g_{1}(s)=\inf \{u<0: L(u)>s\}, s>0$, and $g_{2}(s)=\inf \{u<0:-R(-u)>s\}, s>0$, then $g_{1}, g_{2} \in \Psi$. With these $g_{1}, g_{2}$, the characteristic function of $V_{0}\left(g_{1}, g_{2}, \sigma\right)$ satisfies (3.7).

Now fix the functions $M_{1}$ and $M_{2}$ in Theorem 2.1 and $g_{j}(s)=$ $-M_{j}(s) s^{-1 / p}, j=1,2$. Let $L$ and $R$ be the functions in Lévy's approach corresponding to $g_{1}$ and $g_{2}$, respectively. Let $a_{n}=k_{n^{*}(1 / n)} / n$. Then $1 \leqslant a_{n} \leqslant c_{0}<\infty$. The $L$-function corresponding to the $g_{1}$-function $-M_{1}\left(a_{n} s\right) / s^{1 / p}=-M_{1}\left(\left(k_{n^{*}(1 / n)} / n\right) s\right) / s^{1 / p}$ is $\left(1 / a_{n}\right) L\left(u\left(1 / a_{n}\right)^{1 / p}\right)$. The connection between $M_{2}$ and $R$ is similar.

We shall write $\int \cdot d J(u)$ instead of $\int_{-\infty}^{0} \cdot d L(u)+\int_{0}^{\infty} \cdot d R(u)$.

So the characteristic function of $Y_{n}(1)$ is (compare with (2.12))

$\phi_{n}^{1}(x)=\exp \left\{\int\left(e^{i x u}-1-\frac{i x u}{1+u^{2}}\right) d\left[\frac{1}{a_{n}} J\left(u\left(\frac{1}{a_{n}}\right)^{1 / p}\right)\right]\right\} \exp \left\{i x \theta\left(a_{n}\right)\right\}$

where

$$
\begin{aligned}
a_{n}= & \frac{k_{n^{*}(1 / n)}}{n}, \quad \theta\left(a_{n}\right)=\theta_{1}\left(a_{n}\right)-\theta_{2}\left(a_{n}\right), \\
\theta_{j}\left(a_{n}\right)= & \int_{0}^{1} \frac{-M_{j}\left(s a_{n}\right) s^{-1 / p}}{1+\left(M_{j}\left(s a_{n}\right) s^{-1 / p}\right)^{2}} d s-\int_{1}^{\infty} \frac{\left(-M_{j}\left(s a_{n}\right) s^{-1 / p}\right)^{3}}{1+\left(M_{j}\left(s a_{n}\right) s^{-1 / p}\right)^{2}} d s \\
= & \frac{1}{a_{n}}\left[\int_{0}^{a_{n}} \frac{-M_{j}(v)\left(v / a_{n}\right)^{-1 / p}}{1+\left(M_{j}(v)\left(v / a_{n}\right)^{-1 / p}\right)^{2}} d v\right. \\
& \left.\quad-\int_{a_{n}}^{\infty} \frac{\left(-M_{j}(v)\left(v / a_{n}\right)^{-1 / p}\right)^{3}}{1+\left(M_{j}(v)\left(v / a_{n}\right)^{-1 / p}\right)^{2}} d v\right], \quad j=1,2 .
\end{aligned}
$$

Finally, we obtain another version of Lévy's description. Let $H: \mathbf{R} \rightarrow \mathbf{R}$ be a truncation function (i.e., $H$ is bounded, $H(u)=u$ in a neighborhood of the origin and the support of $H$ is compact). Then

$$
\begin{aligned}
\phi_{n}^{1}(x)= & \exp \left\{\int\left(e^{i x v}-1-i x H(v)\right) d\left[\frac{1}{a_{n}} J\left(v\left(\frac{1}{a_{n}}\right)^{1 / p}\right)\right]\right\} \\
& \times \exp \left\{i x\left(\theta\left(a_{n}\right)+\kappa\left(a_{n}\right)\right)\right\} .
\end{aligned}
$$

Here

$$
\kappa\left(a_{n}\right)=\int\left(H(v)-\frac{v}{1+v^{2}}\right) d\left[\frac{1}{a_{n}} J\left(v\left(\frac{1}{a_{n}}\right)^{1 / p}\right)\right] .
$$

We see that the sequences $\theta\left(a_{n}\right)$ and $\kappa\left(a_{n}\right), n=1,2, \ldots$, are bounded. 
4. Approximation and tightness in space $\mathbf{D}[0,1]$. In this section some general facts on merging probability measures are listed, moreover, tightness of our sequences of probabilities is proved.

Let $\mathbf{H}$ be a complete separable metric space. We shall consider probability measures on the Borel sets of $\mathbf{H}$. Let $\varrho$ denote the Lévy-Prokhorov metric of probability measures (see [9]).

D e f i n ition 4.1. We say that two sequences $\left\{\mu_{n}\right\}$ and $\left\{\nu_{n}\right\}$ of probability measures on $\mathbf{H}$ are merging if $\lim _{n \rightarrow \infty} \varrho\left(\mu_{n}, \nu_{n}\right)=0$.

R e m a r k 4.1 [8, Theorem 1]. $\lim _{n \rightarrow \infty} \varrho\left(\mu_{n}, \nu_{n}\right)=0$ if and only if

$$
\int f d \mu_{n}-\int f d \nu_{n} \rightarrow 0
$$

for each bounded and uniformly continuous function $f: \mathbf{H} \rightarrow \mathbf{R}$.

If at least one of the two sequences of measures are relatively compact, then the situation is quite simple.

$\mathrm{R}$ e $\mathrm{m}$ a r k 4.2 (see $\left[8\right.$, p. 87]). Let $\left\{\mu_{n}\right\}$ be relatively compact and assume that $\lim _{n \rightarrow \infty} \varrho\left(\mu_{n}, \nu_{n}\right)=0$. Then $\left\{\nu_{n}\right\}$ is also relatively compact.

To see it choose a subsequence $\left\{\nu_{n^{\prime}}\right\}$. Then we can choose a further subsequence $\left\{n^{\prime \prime}\right\}$ of $\left\{n^{\prime}\right\}$ such that $\left\{\mu_{n^{\prime \prime}}\right\}$ is convergent. Then $\left\{\nu_{n^{\prime \prime}}\right\}$ converges to the same limit. That is, $\left\{\nu_{n}\right\}$ is relatively compact.

$\mathrm{R} \mathrm{e} \mathrm{m}$ a r k 4.3 (see $[8, \mathrm{p} .87]$ ). Let $\left\{\mu_{n}\right\}$ be relatively compact. Then $\lim _{n \rightarrow \infty} \varrho\left(\mu_{n}, \nu_{n}\right)=0$ if and only if (4.1) is satisfied for each bounded and continuous function $f: \mathbf{H} \rightarrow \mathbf{R}$.

To see it first observe that the proposition is true if $\mu_{n} \equiv \mu$ (see the usual theory in [2]). Now both directions can be obtained using indirect proofs, and convergent subsequences.

Lemma 4.1. Assume that $\left\{\mu_{n}\right\}$ and $\left\{\nu_{n}\right\}$ are tight sequences of probability measures on $\mathbf{D}[0,1]$, moreover

$$
\begin{aligned}
& \text { the distances of the finite dimensional distributions } \\
& \text { of } \mu_{n} \text { and } \nu_{n} \text { converge to } 0 .
\end{aligned}
$$

Then $\lim _{n \rightarrow \infty} \varrho\left(\mu_{n}, \nu_{n}\right)=0$.

P r o o f. Assume that $\lim _{n \rightarrow \infty} \varrho\left(\mu_{n}, \nu_{n}\right)=0$ is not satisfied. Then there exist an $\varepsilon>0$ and a subsequence $\left\{m_{n}\right\}$ such that $\varrho\left(\mu_{m_{n}}, \nu_{m_{n}}\right) \geqslant \varepsilon$ for every $n$. Since $\left\{\mu_{n}\right\}$ and $\left\{\nu_{n}\right\}$ are relative compact, we can choose a further subsequence $\left\{m_{n}^{\prime}\right\}$ of $\left\{m_{n}\right\}$ such that $\mu_{m_{n}^{\prime}} \stackrel{d}{\longrightarrow} \mu$ and $\nu_{m_{n}^{\prime}} \stackrel{d}{\longrightarrow} \nu$, say. Then $\varrho(\mu, \nu) \geqslant \varepsilon$.

Now consider the finite dimensional distributions. We list some known facts. Let $t_{1}, \ldots, t_{k}$ be points in $[0,1]$. The projection

$$
\pi_{t_{1}, \ldots, t_{k}}: \mathbf{D}[0,1] \rightarrow \mathbf{R}^{k}
$$


is defined by $\pi_{t_{1}, \ldots, t_{k}}(x)=\left(x\left(t_{1}\right), \ldots, x\left(t_{k}\right)\right), x \in \mathbf{D}[0,1]$.

Let $T$ be a subset of $[0,1]$. Let $\mathscr{F}_{T}$ denote the class of sets $\pi_{t_{1}, \ldots, t_{k}}^{-1} H$, where $k$ is arbitrary, the $t_{i}$ are arbitrary points of $T$, and $H$ is arbitrary $k$ dimensional Borel set. By Theorem 14.5 of [2], if $T$ contains 1 and is dense in $[0,1]$, then $\mathscr{F}_{T}$ generates $\mathscr{D}$ (the class of the Borel sets of $\mathbf{D}[0,1]$ ).

For any probability measure $P$ on $\mathbf{D}[0,1]$, let $T_{P}$ consists of those $t$ in $[0,1]$ for which the projection $\pi_{t}$ is continuous except at points forming a set of $P$-measure 0 . It is known (see $\left[2\right.$, Section 15]) that $T_{P}$ contains 0 and 1 and its complement in $[0,1]$ is at most countable.

If $P_{n}$ and $P$ are probability measures on $\mathbf{D}[0,1]$ and $P_{n} \stackrel{d}{\longrightarrow} P$, then for the finite dimensional distributions we have that $P_{n} \pi_{t_{1}, \ldots, t_{k}}^{-1} \stackrel{d}{\longrightarrow} P \pi_{t_{1}, \ldots, t_{k}}^{-1}$ holds if all the $t_{i}$ lie in $T_{P}$ (see [2, Section 15]).

By Theorem 15.1 of [2], if the family $\left\{P_{n}\right\}$ is tight and $P_{n} \pi_{t_{1}, \ldots, t_{k}}^{-1} \stackrel{d}{\longrightarrow}$ $P \pi_{t_{1}, \ldots, t_{k}}^{-1}$ holds whenever $t_{1}, \ldots, t_{k}$ all lie in $T_{P}$, then $P_{n} \stackrel{d}{\longrightarrow} P$.

Now we can finish the proof in the following way. From $\mu_{m_{n}^{\prime}} \stackrel{d}{\longrightarrow} \mu$ it follows that $\mu_{m_{n}^{\prime}} \pi_{t_{1}, \ldots, t_{k}}^{-1} \stackrel{d}{\longrightarrow} \mu \pi_{t_{1}, \ldots, t_{k}}^{-1}$ whenever $t_{1}, \ldots, t_{k}$ all lie in $T_{\mu}$. This fact and

$$
\varrho\left(\mu_{n} \pi_{t_{1}, \ldots, t_{k}}^{-1}, \nu_{n} \pi_{t_{1}, \ldots, t_{k}}^{-1}\right) \rightarrow 0
$$

imply that

$$
\nu_{m_{n}^{\prime}} \pi_{t_{1}, \ldots, t_{k}}^{-1} \stackrel{d}{\longrightarrow} \mu \pi_{t_{1}, \ldots, t_{k}}^{-1}
$$

whenever $t_{1}, \ldots, t_{k}$ all lie in $T_{\mu}$. However, $\nu_{m_{n}^{\prime}} \stackrel{d}{\longrightarrow} \nu$ implies

$$
\nu_{m_{n}^{\prime}} \pi_{t_{1}, \ldots, t_{k}}^{-1} \stackrel{d}{\longrightarrow} \nu \pi_{t_{1}, \ldots, t_{k}}^{-1}
$$

whenever $t_{1}, \ldots, t_{k}$ all lie in $T_{\nu}$. Now, by (4.3) and (4.4),

$$
\mu \pi_{t_{1}, \ldots, t_{k}}^{-1}=\nu \pi_{t_{1}, \ldots, t_{k}}^{-1}
$$

if $t_{1}, \ldots, t_{k} \in T_{\nu} \cap T_{\mu}=T$. Now, $\mathscr{F}_{T}$ is an algebra of sets and, by the above equality, $\mu$ and $\nu$ coincide on $\mathscr{F}_{T}$. Since $T$ is dense in $[0,1]$ and it contains 1 , $\mathscr{F}_{T}$ generates $\mathscr{D}$. So $\mu$ and $\nu$ coincide on $\mathscr{D}$. It contradicts to $\varrho(\mu, \nu) \geqslant \varepsilon$. Lemma 4.1 is proved.

$\mathrm{R} \mathrm{e} \mathrm{m} \mathrm{a} \mathrm{r} \mathrm{k} \mathrm{4.4.} \mathrm{Let}\left\{\mu_{n}\right\}$ and $\left\{\nu_{n}\right\}$ be sequences of probability measures on $\mathbf{C}[0,1]$. Assume that $\left\{\mu_{n}\right\}$ is tight. Then $\lim _{n \rightarrow \infty} \varrho\left(\mu_{n}, \nu_{n}\right)=0$ if and only if $\left\{\nu_{n}\right\}$ is tight and (4.2) is satisfied.

To see it first we remark that $\pi_{t_{1}, \ldots, t_{k}}$ is continuous for every $t_{1}, \ldots, t_{k} \in$ $[0,1]$ (see $\left[2\right.$, Section 3]). So, for any probability measures $P, P_{1}, P_{2}, \ldots$ the convergence $P_{n} \stackrel{d}{\longrightarrow} P$ implies for the finite dimensional distributions that $P_{n} \pi_{t_{1}, \ldots, t_{k}}^{-1} \stackrel{d}{\longrightarrow} P \pi_{t_{1}, \ldots, t_{k}}^{-1}$ for every $t_{1}, \ldots, t_{k} \in[0,1]$. Therefore the proof of Lemma 4.1 implies one direction. 
To obtain the other direction let $\lim _{n \rightarrow \infty} \varrho\left(\mu_{n}, \nu_{n}\right)=0$. Then Remark 4.2 implies tightness of $\left\{\nu_{n}\right\}$. Now assume that (4.2) is not satisfied. Then there exist $t_{1}, \ldots, t_{k} \in[0,1]$ and a subsequence $n^{\prime}$ such that

$$
\varrho\left(\mu_{n^{\prime}} \pi_{t_{1}, \ldots, t_{k}}^{-1}, \nu_{n^{\prime}} \pi_{t_{1}, \ldots, t_{k}}^{-1}\right) \geqslant \varepsilon>0 .
$$

However, there exists a further subsequence $\left\{n^{\prime \prime}\right\}$ of $\left\{n^{\prime}\right\}$ such that $\mu_{n^{\prime \prime}} \rightarrow \mu$ and $\nu_{n^{\prime \prime}} \rightarrow \mu$. Their finite dimensional distributions also converge. It is a contradiction.

Now we turn to the proof of tightness of $Y_{n}(t)$. We shall use the notation of [14]. A process $Y(t), t \geqslant 0$, is called a process with stationary independent increments, if it is adapted to the filtration $\mathscr{F}_{t}$, it is càdlàg, $Y(0)=0, Y(t)-$ $Y(s)$ is independent of $\mathscr{F}_{s}(0 \leqslant s \leqslant t)$, and the distribution of $Y(t)-Y(s)$ depends only on the difference $t-s$. (We shall consider $\mathscr{F}_{t}$ as the $\sigma$-field generated by $\{Y(s): s \leqslant t\}$.)

Let $Y_{n}(t), t \geqslant 0, n=0,1,2, \ldots$, be a sequence of processes with stationary independent increments with characteristic functions

$$
\mathbf{E} e^{i x Y_{n}(t)}=\exp \left\{t\left[i x b_{n}-\frac{\sigma_{n}^{2} x^{2}}{2}+\int\left(e^{i x v}-1-i x H(v)\right) d K_{n}(v)\right]\right\} .
$$

Here $H$ is a truncation function that we choose to be continuous. Moreover, for each $n$ : $b_{n} \in \mathbf{R}, \sigma_{n} \geqslant 0, K_{n}$ is a positive measure on $\mathbf{R}$ that integrates $\min \left\{x^{2}, 1\right\}$ and satisfies $K_{n}(0)=0$.

By [14, Chap. VII, Corollary 3.6], we have the following criterion of convergence. Let $Y_{n}(t), t \geqslant 0, n=0,1,2, \ldots$, be a sequence of processes with stationary independent increments with characteristics $b_{n} t, \sigma_{n}^{2} t, d t K_{n}(d x)$. Then there is equivalence between the following three statements:

(a) $Y_{n} \stackrel{d}{\longrightarrow} Y_{0}$;

(b) $Y_{n}(1) \stackrel{d}{\longrightarrow} Y_{0}(1)$;

(c) $b_{n} \rightarrow b_{0}, \tilde{c}_{n}=\sigma_{n}^{2}+\int H^{2}(x) d K_{n}(x) \rightarrow \sigma_{0}^{2}+\int H^{2}(x) d K_{0}(x)=$ $\tilde{c}_{0}, \int g(x) d K_{n}(x) \rightarrow \int g(x) d K_{0}(x)$ for each continuous bounded function $g: \mathbf{R} \rightarrow \mathbf{R}$ which is 0 around 0 and has a limit at infinity.

Lemma 4.2. Let the sequence $Y_{n}, n=1,2, \ldots$, of processes with stationary independent increments be defined by (2.12). Then the sequence of measures $\mathscr{L}_{Y_{n}}, n=1,2, \ldots$, is tight in $\mathbf{D}[0,1]$.

P r o o f. We shall apply the above facts from the theory of processes with stationary independent increments. Let $Y_{n}$ be defined by (2.12). Then, by $(3.8), b_{n}=\theta\left(a_{n}\right)+\kappa\left(a_{n}\right), \sigma_{n}=0, K_{n}(\cdot)=\left(1 / a_{n}\right) J\left(\left(1 / a_{n}\right)^{1 / p} \cdot\right)$.

We have to prove that $\mathscr{L}_{Y_{n}}, n=1,2, \ldots$, is relatively compact. Consider an arbitrary subsequence. We have $1 \leqslant a_{n} \leqslant c_{0}<\infty$. Therefore our subsequence contains a further subsequence $n^{\prime}$ such that $a_{n^{\prime}} \rightarrow a_{0}$, where $1 \leqslant a_{0} \leqslant c_{0}<\infty$. It is easy to see that the sequence $b_{n^{\prime}}$ is convergent. 
Moreover, using the properties of $H$ and $g$, we obtain

$$
\begin{gathered}
\tilde{c}_{n^{\prime}}=\int H^{2}(x) d\left[\frac{1}{a_{n}^{\prime}} J\left(\left(\frac{1}{a_{n^{\prime}}}\right)^{1 / p} x\right)\right] \rightarrow \int H^{2}(x) d\left[\frac{1}{a_{0}} J\left(\left(\frac{1}{a_{0}}\right)^{1 / p} x\right)\right]=\tilde{c}_{0}, \\
\int g(x) d\left[\frac{1}{a_{n}^{\prime}} J\left(\left(\frac{1}{a_{n^{\prime}}}\right)^{1 / p} x\right)\right] \rightarrow \int g(x) d\left[\frac{1}{a_{0}} J\left(\left(\frac{1}{a_{0}}\right)^{1 / p} x\right)\right] .
\end{gathered}
$$

Therefore the subsequence $\mathscr{L}_{Y_{n^{\prime}}}$ is convergent. Lemma 4.2 is proved.

Lemma 4.3. Let the sequence of processes $Z_{n}(t)$ be defined by (2.11). Then the sequence of measures $\mathscr{L}_{Z_{n}}, n=1,2, \ldots$, is tight in $\mathbf{D}[0,1]$.

P r o o f. Introduce the auxiliary sequence $S_{n}^{\prime}=\left(1 / B_{n}\right) \sum_{i=1}^{n} X_{i}-A_{n}^{\prime}$, where

$$
A_{n 1}^{\prime}=\frac{1}{B_{n}} \mathbf{E} X I_{\left\{|X| \leqslant B_{n}\right\}}, \quad A_{n k}^{\prime}=k A_{n 1}^{\prime}, \quad k=1, \ldots, n, \quad A_{n}^{\prime}=A_{n n}^{\prime},
$$

for $n=1,2, \ldots$.

Consider the random processes

$$
Z_{n}^{\prime}(t)=\frac{1}{B_{n}} \sum_{i=1}^{[n t]} X_{i}-[n t] A_{n 1}^{\prime}=Z_{n}(t)+[n t]\left(A_{n 1}-A_{n 1}^{\prime}\right), \quad t \in[0,1] .
$$

We shall prove the tightness of the distribution family $\left\{\mathscr{L}_{Z_{n}^{\prime}}: n \in \mathbf{N}\right\}$.

We denote by $L^{0}$ the space of all random variables endowed with the topology of convergence in probability. By Theorem 1 of [7], the sequence $\left\{S_{n}\right\}_{n=1}^{\infty}$ is bounded in $L^{0}$.

By [18] (see the proof of Theorem 3 therein)

$$
\max \left\{\left|Q_{+}(s)\right|, Q(1-s)\right\} \leqslant C s^{-1 / p} l(s),
$$

for $s>0$ small enough, where $C<\infty$.

We need the following facts. For $a>0$ we have

$$
\begin{array}{ll}
\text { if } a>Q(1-s), & \text { then } 1-F(a) \leqslant s, \\
\text { if }-a<Q_{+}(s), & \text { then } F_{-}(-a) \leqslant s,
\end{array}
$$

where $F_{-}$is the left-continuous version of $F$.

Let $\bar{F}(x)=\mathbf{P}(|X|>x)=1-F(x)+F_{-}(-x)$, for $x>0$. Then, using basic properties of slowly varying functions (see [3, Chap. 1]), we deduce from (4.5) and (4.6) that

$$
\sup _{n \in \mathbf{N}} n \mathbf{P}\left\{\left|\frac{X}{B_{n}}\right|>1\right\}=\sup _{n \in \mathbf{N}} n \bar{F}\left(B_{n}\right)=C_{1}<\infty .
$$


Now let $U_{n}=\left(1 / B_{n}\right) \sum_{i=1}^{n}\left|X_{i}\right| I_{\left\{\left|X_{i}\right|>B_{n}\right\}}, n=1,2, \ldots$ Integrating by parts, we obtain

$$
\mathbf{P}\left(\left|U_{n}\right|>K\right) \leqslant \frac{n \bar{F}\left(B_{n}\right)}{K}+\int_{1 / K}^{1} n \bar{F}\left(K B_{n} x\right) d x .
$$

Then, using known facts on regularly varying functions (see Potter's theorem in [3, Chap. 1]), (4.5), and (4.6), we can see that $\int_{1 / K}^{1} n \bar{F}\left(K B_{n} x\right) d x<\varepsilon$ if $K>K_{\varepsilon}$. This fact, (4.8), and (4.7) imply that $U_{n}$ is bounded in probability. Therefore $U_{n}^{\prime}=\left(1 / B_{n}\right) \sum_{i=1}^{n} X_{i} I_{\left\{\left|X_{i}\right|>B_{n}\right\}}, n=1,2, \ldots$, is also bounded in probability.

Let $V_{n}=\left(1 / B_{n}\right) \sum_{i=1}^{n} X_{i} I_{\left\{\left|X_{i}\right| \leqslant B_{n}\right\}}, n=1,2, \ldots$ Then $V_{n}-A_{n}=$ $S_{n}-U_{n}^{\prime}, n=1,2, \ldots$, is also bounded in probability. Using this fact and applying the same method (symmetrization, Lévy's inequality, and stopping times) as in the proof of Theorem 10.1.1 in [4], we can prove that

$$
\sup _{n \in \mathbf{N}} n \mathbf{D}\left(\frac{X}{B_{n}} I_{\left\{|X| \leqslant B_{n}\right\}}\right)^{2}=C_{2}<\infty .
$$

Using this fact and Chebyshev's inequality, we can see that the sequence $\left\{S_{n}^{\prime}\right\}$ is bounded in probability. Since $\left\{S_{n}\right\}$ and $\left\{S_{n}^{\prime}\right\}$ are bounded in probability, the sequence $\left\{A_{n}-A_{n}^{\prime}\right\}$ is bounded.

Now, turn to the tightness of the distribution family $\left\{\mathscr{L}_{Z_{n}^{\prime}}: n \in \mathbf{N}\right\}$. To this end we apply Theorem 15.3 of [2]. Let $0<\varepsilon<1$ and $0<u<1$. We have

$$
\begin{aligned}
\mathbf{P}\left\{\sup _{0 \leqslant t \leqslant u}\left|Z_{n}^{\prime}(t)\right|>\varepsilon\right\} \\
\leqslant \mathbf{P}\left\{\sup _{0 \leqslant t \leqslant u}\left|\frac{1}{B_{n}} \sum_{i=1}^{[n t]}\left(X_{i} I_{\left\{\left|X_{i}\right| \leqslant B_{n}\right\}}-\mathbf{E} X_{i} I_{\left\{\left|X_{i}\right| \leqslant B_{n}\right\}}\right)\right|>\frac{\varepsilon}{2}\right\} \\
\quad+[n u] \mathbf{P}\left\{|X|>B_{n}\right\} .
\end{aligned}
$$

Now, applying Kolmogorov's inequality, (4.10), (4.7), and (4.9), we obtain

$$
\lim _{u \rightarrow 0} \limsup _{n \rightarrow \infty}\left(\mathbf{P}\left\{\sup _{0 \leqslant t \leqslant u}\left|Z_{n}^{\prime}(t)\right|>\varepsilon\right\}+\mathbf{P}\left\{\sup _{1-u \leqslant t \leqslant 1}\left|Z_{n}^{\prime}(1)-Z_{n}^{\prime}(t)\right|>\varepsilon\right\}\right)=0 .
$$

Using the inequality in [11, Chap. 9, Section 6], (4.10), Kolmogorov's inequality, (4.7), and (4.9), we obtain

$$
\begin{aligned}
& \lim _{m \rightarrow \infty} \limsup _{n \rightarrow \infty} \sum_{k=1}^{m} \mathbf{P}\left\{\operatorname { s u p } _ { \frac { k - 1 } { m } \leqslant t _ { 1 } \leqslant t _ { 2 } \leqslant t _ { 3 } < \frac { k } { m } } \operatorname { m i n } \left\{\left|Z_{n}^{\prime}\left(t_{2}\right)-Z_{n}^{\prime}\left(t_{1}\right)\right|,\right.\right. \\
& \left.\left.\left|Z_{n}^{\prime}\left(t_{3}\right)-Z_{n}^{\prime}\left(t_{2}\right)\right|\right\}>\varepsilon\right\} \\
& \leqslant \lim _{m \rightarrow \infty} \limsup _{n \rightarrow \infty} \sum_{k=1}^{m}\left(\mathbf{P}\left\{\sup _{\frac{k-1}{m} \leqslant t<\frac{k}{m}}\left|Z_{n}^{\prime}\left(\frac{k}{m}\right)-Z_{n}^{\prime}(t)\right|>\frac{\varepsilon}{4}\right\}\right)^{2}
\end{aligned}
$$




$$
\begin{gathered}
\leqslant \lim _{m \rightarrow \infty} \limsup _{n \rightarrow \infty} \max _{1 \leqslant k \leqslant m}\left\{\frac { C ( [ \frac { k } { m } n ] - [ \frac { k - 1 } { m } n ] ) } { \varepsilon ^ { 2 } } \left\{\mathbf{E}\left(\frac{X}{B_{n}} I_{\left\{|X| \leqslant B_{n}\right\}}-A_{n 1}^{\prime}\right)^{2}\right.\right. \\
\left.\left.+\mathbf{P}\left\{|X|>B_{n}\right\}\right\}\right\} \\
\times \sum_{k=1}^{m} \frac{C\left(\left[\frac{k}{m} n\right]-\left[\frac{k-1}{m} n\right]\right)}{\varepsilon^{2}}\left\{\mathbf{E}\left\{\frac{X}{B_{n}} I_{\left\{|X| \leqslant B_{n}\right\}}-A_{n 1}^{\prime}\right\}^{2}\right. \\
\left.+\mathbf{P}\left\{|X|>B_{n}\right\}\right\}=0
\end{gathered}
$$

Let $Z_{n}^{\prime}(t)=Z_{1 n}^{\prime}(t)+Z_{2 n}^{\prime}(t), t \in[0,1]$, where

$$
Z_{1 n}^{\prime}(t)=\frac{1}{B_{n}} \sum_{i=1}^{[n t]} X_{i} I_{\left\{\left|X_{i}\right| \leqslant B_{n}\right\}}-[n t] A_{n 1}^{\prime}, \quad Z_{2 n}^{\prime}(t)=\frac{1}{B_{n}} \sum_{i=1}^{[n t]} X_{i} I_{\left\{\left|X_{i}\right|>B_{n}\right\}},
$$

for $t \in[0,1]$. By Kolmogorov's inequality and (4.9), we have

$$
\sup _{n \in \mathbf{N}} \mathbf{P}\left\{\left\|Z_{1 n}^{\prime}\right\|_{\infty}>K\right\} \leqslant \sup _{n \in \mathbf{N}} \frac{n \mathbf{E}\left(\left(1 / B_{n}\right) X I_{\left\{|X| \leqslant B_{n}\right\}}-A_{n 1}^{\prime}\right)^{2}}{K^{2}} \leqslant \frac{C_{2}}{K^{2}} \rightarrow 0
$$

as $K \rightarrow \infty$. We have already proved, that the family of random variables $\left\{U_{n}\right\}$ is bounded in $L^{0}$. Therefore

$$
\sup _{n \in \mathbf{N}} \mathbf{P}\left\{\left\|Z_{2 n}^{\prime}\right\|_{\infty}>K\right\} \leqslant \sup _{n \in \mathbf{N}} \mathbf{P}\left\{\left|U_{n}\right|>K\right\} \rightarrow 0 \quad \text { as } K \rightarrow \infty .
$$

From (4.13) and (4.14) we obtain

$$
\sup _{n \in \mathbf{N}} \mathbf{P}\left\{\left\|Z_{n}^{\prime}\right\|_{\infty}>K\right\} \rightarrow 0 \quad \text { as } K \rightarrow \infty .
$$

Now, by Theorem 15.3 in [2], relations (4.11), (4.12), and (4.15) imply the tightness of the distribution family $\left\{\mathscr{L}_{Z_{n}^{\prime}}: n \in \mathbf{N}\right\}$. Therefore for any $\varepsilon>0$ there exists a compact set $K_{\varepsilon}^{\prime} \subset \mathbf{D}[0,1]$ such that $\mathscr{L}_{Z_{n}^{\prime}}\left(K_{\varepsilon}^{\prime}\right)>1-\varepsilon$ for all $n \in \mathbf{N}$.

Now, we prove the tightness of $\left\{\mathscr{L}_{Z_{n}}: n \in \mathbf{N}\right\}$. Let $c_{n}=A_{n}^{\prime}-A_{n}$. We have already proved that $\left\{c_{n}\right\}$ is bounded. Consider the sequence of functions

$$
f_{n}(t)=[n t]\left(A_{n 1}^{\prime}-A_{n 1}\right)=c_{n} \frac{[n t]}{n}, \quad t \in[0,1] .
$$

Let $K_{1}=\left\{f_{n}: n=1,2, \ldots\right\}$. Let $\Lambda$ denote the class of strictly increasing, continuous mappings of $[0,1]$ onto itself (i.e., $\Lambda$ is the set of functions used to define the topology of $\mathbf{D}[0,1]$, see $[2])$. Let $\lambda \in \Lambda$. Since $t-(1 / n) \leqslant[n t] / n \leqslant$ $t$, we have

$$
\left|f_{n}(\lambda(t))-c_{n} \lambda(t)\right| \leqslant \frac{\left|c_{n}\right|}{n} .
$$


From this inequality and from the boundedness of $\left\{c_{n}\right\}$ we obtain that from any infinite sequence from $K_{1}$ we can select a subsequence $\left\{f_{k_{n}}\right\}$ such that

$$
\lim _{n \rightarrow \infty} f_{k_{n}}\left(\lambda_{n}(t)\right)=c t
$$

uniformly in $t$ for an arbitrary sequence $\left\{\lambda_{n}(\cdot)\right\}$ from $\Lambda$ with $\lim _{n \rightarrow \infty} \lambda_{n}(t)=$ $t$ uniformly in $t$.

Now we can prove that $K_{\varepsilon}=K_{\varepsilon}^{\prime}+K_{1}$ is a compact set in $\mathbf{D}[0,1]$. Consider a sequence $g_{n}+h_{n}$ from $K_{\varepsilon}^{\prime}+K_{1}$. Since $K_{\varepsilon}^{\prime}$ is compact, we can choose a convergent subsequence $g_{k_{n}}$. That is (see [2, Section 14]), there exist functions $\lambda_{k_{n}}$ in $\Lambda$ such that $\lim _{n \rightarrow \infty} g_{k_{n}}\left(\lambda_{k_{n}}(t)\right)=g(t)$ and $\lim _{n \rightarrow \infty} \lambda_{k_{n}}(t)=$ $t$ uniformly in $t$. Thus, by (4.16), there exists a further subsequence such that $\lim _{n \rightarrow \infty}\left[g_{k_{n}^{\prime}}\left(\lambda_{k_{n}^{\prime}}(t)\right)+h_{k_{n}^{\prime}}\left(\lambda_{k_{n}^{\prime}}(t)\right)\right]=g(t)+c t$ uniformly in $t$. It means that $g_{k_{n}^{\prime}}(t)+h_{k_{n}^{\prime}}(t) \rightarrow g(t)+c t$ in Skorokhod's topology.

Finally, for all $n \in \mathbf{N}$ we have

$$
\mathscr{L}_{Z_{n}}\left(\mathbf{D}[0,1] \backslash K_{\varepsilon}\right) \leqslant \mathscr{L}_{Z_{n}^{\prime}}\left(\mathbf{D}[0,1] \backslash K_{\varepsilon}^{\prime}\right)<\varepsilon .
$$

Lemma 4.3 is proved.

Acknowledgments. I am grateful to Prof. I. Berkes for proposing the topic. I thank Prof. A. Rackauskas for reference [8].

\section{REFERENCES}

1. Berkes I., Csáki E., Csörgő S., Megyesi S. Almost sure limit theorems for sums and maxima from the domain of geometric partial attraction of semistable laws. — Limit Theorems in Probability and Statistics, v. I. Ed. by I. Berkes, E. Csáki, and M. Csörgö, Budapest: János Bolyai Math. Soc., 2002, p. 133-157.

2. Биллингсли П. Сходимость вероятностных мер. М.: Наука, 1977, 351 с.

3. Bingham N. H., Goldie C. M., Teugels J. L. Regular Variation. Cambridge: Cambridge University Press, 1987, $491 \mathrm{p}$.

4. Chow Y.S., Teicher H. Probability Theory. Independence, Interchangeability, Martingales. New York-Heidelberg: Springer-Verlag, 1978, 455 p.

5. Csörgö S. A probabilistic approach to domains of partial attraction. - Adv. Appl. Math., 1990, v. 11, № 3, p. 282-327.

6. Csörgő S., Haeusler E., Mason D. M. A probabilistic approach to the asymptotic distribution of sums of independent, identically distributed random variables. - Adv. Appl. Math., 1988, v. 9, № 3, p. 259-333.

7. Csörgö S., Megyesi Z. Merging to semistable laws. - Теория вероятн. и ее примен., 2002 , т. 47 , в. 1 , с. $90-109$.

8. Davydov $Y$., Rotar $V$. On asymptotic proximity of distributions. - J. Theoret. Probab., 2009, v. 22, № 1, p. 82-98.

9. Dudley R.M. Real Analysis and Probability. Pacific Grove: Wadsworth \& Brooks/Cole, 1989, 436 p.

10. Fazekas I., Chuprunov A. An almost sure functional limit theorem for the domain of geometric partial attraction of semistable laws. - J. Theoret. Probab., 2007, v. 20, № 2 , p. 339-353.

11. Гихман И.И., Скороход А.В. Введение в теорию случайных процессов. М.: Наука, 1965, 655 с. 
12. Гнеденко Б.В., Колмогоров А.Н. Предельные распределения для сумм независимых случайных величин. М.-Л.: Гостехиздат, 1949, 264 с.

13. Гриневич И. В., Хохлов Ю. С. Области притяжения полуустойчивых законов. Теория вероятн. и ее примен., 1995, т. 40, в. 2, с. 417-422.

14. Жакод Ж., Ширяев А.Н. Предельные теоремы для случайных процессов, т. 1, 2. М.: Наука, 1994, 542 с., 366 с.

15. Kevei P., Csörgö S. Merging of linear combinations to semistable laws. - J. Theoret. Probab., 2009, v. 22, № 3, p. 772-790.

16. Круглов В.М. Об одном расширении класса устойчивых распределений. Теория вероятн. и ее примен., 1972, т. 17, в. 4, с. $723-732$.

17. Lévy $P$. Théorie de l'addition des variables aléatoires. Paris: Gauthier-Villars, 1937.

18. Megyesi $Z$. A probabilistic approach to semistable laws and their domains of partial attraction. - Acta Sci. Math. (Szeged), 2000, v. 66, № 1-2, p. 403-434.

19. Прохоров Ю. В. Сходимость случайных процессов и предельные теоремы теории вероятностей. - Теория вероятн. и ее примен., 1956, т. 1, в. 2, с. 177-238.

20. Sato K. Lévy Processes and Infinitely Divisible Distributions. Cambridge: Cambridge University Press, 1999, 486 p.

21. Скороход A.В. Предельные теоремы для случайных процессов. - Теория вероятн. и ее примен., 1956, т. 1, в. 3, с. 289-319.

Поступила в редакцию

18.VI.2010

Исправленный вариант

1.VII.2011 